\title{
VEGFR and EGFR inhibition increases epithelial cellular characteristics and chemotherapy sensitivity in mesenchymal bladder cancer cells
}

\author{
YUAN LI ${ }^{1,2}$, XIAOPING YANG ${ }^{1}$, LIH-JEN SU ${ }^{1}$ and THOMAS W. FLAIG ${ }^{1}$ \\ ${ }^{1}$ Division of Medical Oncology, Department of Medicine, University of Colorado Denver \\ School of Medicine, Aurora, CO 80045, USA
}

Received March 24, 2010; Accepted May 19, 2010

DOI: $10.3892 /$ or_00000950

\begin{abstract}
The present study investigated the effect of VEGFR and EGFR inhibition via vandetanib (Zactima ${ }^{\mathrm{TM}}$ ) on epithelialmesenchymal transition (EMT) in bladder cancer. Markers of EMT (EGFR, VEGR, E-cadherin and vimentin) were interogated by Western blotting at baseline and after treatment with EGF, VEGF, vandetanib, cisplatin, or their combination using representative epithelial- and mesenchymal-type human bladder cancer cells. Morphological changes induced by these treatments were examined by microscopy over various time courses. The effect of these changes on cisplatin chemotherapy sensitivity was assessed by MTT assay. RT4 and HTB3 cells had epithelial features while CRL1749 and J82 cells had mesenchymal features. After treatment with EGF, the epithelial-type cells demonstrated increased intercellular separation and pseudopodia, with these changes blocked by vandetanib. In contrast, the mesenchymal cells did not exhibit any morphological changes with the EGF treatment but adopted a clustered/epithelial appearance after the administration of vandetanib. Western blotting shows that treatment of epithelial cells with vandetanib increased the expression of E-cadherin. In comparison, mesenchymal cells demonstrated decreased vimentin expression with the treatment of vandetanib in the presence of EGF and VEGF. Improved growth inhibition was seen in the epithelial cells but not in mesenchymal cells with the concurrent treatment of vandetanib and cisplatin. Sequential treatment of mesenchymal cells with vandetanib followed by cisplatin demonstrated synergy with improved cisplatin activity. The findings offer a novel role of vandetanib
\end{abstract}

Correspondence to: Dr Xiaoping Yang, Division of Medical Oncology, University of Colorado Denver School of Medicine, Mail Stop 8117, P.O. Box 6511, Aurora, CO 80045-0511, USA

E-mail: xiaoping.yang@ucdenver.edu

Present address: ${ }^{2}$ The Department of Urology, XiangYa Hospital, Central South University, Hunan, P.R. China

Key words: bladder cancer, epithelial-mesenchymal transition, chemotherapy, vandetanib on the EMT in bladder cancer, providing insight into EMT in bladder cancer.

\section{Introduction}

Bladder cancer is the 6th most common cancer in the USA, with 68,810 new cases and 14,100 deaths estimated in 2008 (1). Combination chemotherapy regimens produce objective responses; however, the 5-year overall survival rates for patients with locally advanced or metastatic bladder cancer remain disappointingly low at $10-15 \%$ (2). The mechanisms facilitating progression from superficial to invasive disease, and finally to metastatic bladder carcinoma remain largely elusive.

Epithelial-mesenchymal transition (EMT) is a process first observed in embryonic development (3-5). EMT results in the formation of highly motile cells which are critical in early developmental biology. Accumulating evidence suggests that EMT may play an important role in the late stages of tumor progression and metastasis $(6,7)$. During this transition, cells acquire a morphological and functional state that is suitable for migration in the extracellular environment. This allows for the movement required in development and organ formation, but also leads to resistance to anoikis (apoptosis related to cellular detachment from the extracellular membrane) and to traditional chemotherapy when it occurs in cancer $(8,9)$.

Epidermal growth factor receptor (EGFR) and vascular endothelial growth factor receptor (VEGFR) signaling are critical for cell proliferation and cancer progression $(10,11)$. The extracellular signals induced by the epidermal growth factor (EGF) and vascular endothelial growth factor (VEGF) are strongly implicated in both EMT and chemotherapy resistance in human epithelial tumors (12-14). The present study investigates the relationship between EGF, VEGF and EMT in bladder cancer cell lines and on their chemotherapy sensitivity.

Several pathways including NF-кB, AKT/mTOR axis, MAPK, PKC and the AP-1/SMAD are putative actors in EMT, with many of these emerging as potential targets of novel biological agents (15-18). Cytotoxic drug therapy combined with EMT signaling inhibitors (including inhibition 
of upstream pathways), may circumvent the chemotherapeutic resistance of cancers associated with EMT (12).

Vandetanib (Zactima ${ }^{\mathrm{TM}}$ ) is an investigational, once-daily, oral anticancer drug that selectively inhibits VEGFR and EGFR tyrosine kinase activities (19). The effects of vandetanib on bladder cancer have not been studied yet. In our previous study, we showed that low concentration $(1 \mu \mathrm{M})$ of vandetanib exhibited a synergistic effect with cisplatin in human bladder cancer cells (20). No report exists on exploring the effect of vandetanib on EMT. To continue our efforts to investigate the molecular mechanisms of the anticancer effects of vandetanib, the current study was designed to examine the relationship between EMT and exogenous EGF and VEGF in bladder cancer cells. Vandetanib allows for the selective study of the effects of VEGF and EGF on EMT and importantly, the effect of such manipulation on the efficacy of chemotherapy combinations against bladder cancer.

\section{Materials and methods}

Cell culture and reagents. Human bladder cancer cell lines HTB3, J82, RT4, CRL1749 (American Type Culture Collection, Manassas, VA) were grown in OPTI-MEM (Gibco, Grand Island, NY) with $3.75 \%$ fetal bovine serum (FBS) (Gemini, Woodland, CA) and $100 \mu \mathrm{g} / \mathrm{ml}$ streptomycin-100 IU/ $\mathrm{ml}$ penicillin sulfate (Life Technologies, Grand Island, NY). All cell lines were incubated at $37^{\circ} \mathrm{C}$ in $5 \% \mathrm{CO}_{2}$. Before the cells were exposed to exogenous EGF, or VEGF alone or in combination with vandetanib, they were serum-starved $(0.5 \% \mathrm{FBS})$ for $24 \mathrm{~h}$ in 6 -well plates. Vandetanib (from AstraZeneca Pharmaceuticals, Macclesfield, UK) was dissolved in DMSO. Cisplatin (Bedford Laboratories, Bedford, $\mathrm{OH}$ ) was obtained in solution from the University of Colorado Hospital Pharmacy (Aurora, CO). Antibodies to EGFR (Cell Signaling, Beverly, MA) (1:1,000 dilution), VEGFR-2 and B-actin (Santa Cruz Biotechnology, Inc., Santa Cruz, CA) (1:1,000 dilution), and E-cadherin and vimentin (Zymed Laboratories, San Francisco, CA) (1:1,000 dilution) were used for Western blot analysis. Recombinant human VEGFA165 and EGF were purchased from R\&D Systems, Inc. (Minneapolis, MN).

Morphological observation. The cells were cultured with EGF, VEGF, vandetanib or the combination of one of the growth factors plus vandetanib consecutively for $72 \mathrm{~h}$. The concentrations of EGF or VEGF were 5, 10, 20, $40 \mathrm{ng} / \mathrm{ml}$. The concentration of vandetanib was $1.25 \mu \mathrm{M}$ for the RT4 cells and $2.5 \mu \mathrm{M}$ for the CRL1749 cells. The treatment medium was changed daily for each condition. Morphologic characteristics including cell distribution pattern, intercellular space and pseudopodia were observed with a Nikon light microscope at a magnification of x200 and imaged every $24 \mathrm{~h}$ with digital photography.

Combinatorial effects of vandetanib and cisplatin on protein expression and cell viability. The synergistic effects of vandetanib $(0,0.625$ and $2.5 \mu \mathrm{M})$ in combination with cisplatin $(0,1.25,2.5,5$ and $10 \mu \mathrm{M})$ were investigated in selected cell lines, in media containing $3.75 \%$ FBS. The effects of $48 \mathrm{~h}$

$\begin{array}{llll}\text { HTB3 } & \text { J82 } & \text { CRL1749 } & \text { RT4 }\end{array}$

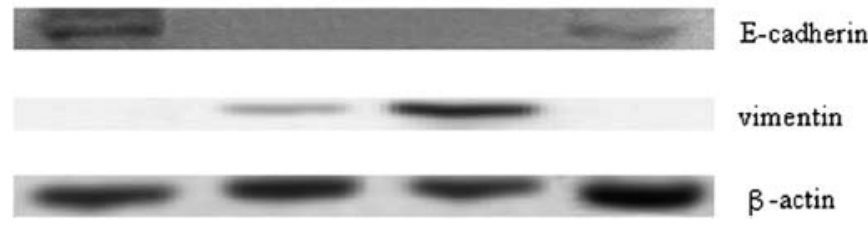

Figure 1. HTB3, J82, CRL1749, and RT4 cells were cultured in medium with $3.75 \%$ FBS. RT4 and HTB3 cells have a complete opposite expression of E-cadherin and vimentin compared with CRL1749 and J82 cells.

of concurrent vandetanib and cisplatin in these cells were evaluated. To further examine the importance of treatment sequence on the observed synergy, RT4 and CRL1749 cells were treated with vandetanib-containing media for $48 \mathrm{~h}$, followed by cisplatin-containing media for an additional $48 \mathrm{~h}$. The sequential treatment with cisplatin followed by vandetanib was also performed. After treatment, the protein expression and cell viability were determined as described below.

Protein characterization. After the specified treatment was completed, the cells were harvested by scraping, washed with PBS and collected by centrifugation. Cell pellets were resuspended in lysis solution $(10 \mathrm{mM}$ Tris, $150 \mathrm{mM} \mathrm{NaCl}$, $1 \%$ Triton $\mathrm{X}-100,1 \mathrm{mM}$ EDTA, $1 \mathrm{mM}$ EGTA, $0.2 \mathrm{mM}$ sodium orthovanadate, $0.5 \%$ NP-40, $0.3 \mathrm{mM}$ PMSF, $10 \mu \mathrm{g} / \mathrm{ml}$ aprotinin). Protein estimation was performed with Pierce protein dye, using a DU 800 Beckman Coulter spectrophotometer (Fullerton, CA). Protein $(50 \mu \mathrm{g})$ was electrophoresed using NuPAGE 4-12\% Bis-Tris Gel (Invitrogen, Carlsbad, CA). The protein was transferred to a nitrocellulose membrane (Invitrogen) using a wet method at $100 \mathrm{~V}$ for $1 \mathrm{~h}$. The membrane was then blocked with $5 \%(\mathrm{w} / \mathrm{v})$ milk powder and placed in a rotator for $1 \mathrm{~h}$ at room temperature. The primary antibody was added in milk powder $(2.5 \% \mathrm{w} / \mathrm{v})$ and allowed to incubate overnight at $4{ }^{\circ} \mathrm{C}$ before being washed with PBS/ $0.05 \%$ Tween-20 three times (15 min each). The appropriate horseradish peroxidase-linked secondary antibody was then added and incubated for an additional $1 \mathrm{~h}$ at room temperature. The membrane was again washed three times for 15 min each, before the addition of Pierce SuperSignal chemiluminescent substrate (Rockford, IL) and then immediately imaged on ChemiDoc (Bio-Rad, Hercules, CA). The protein band densities were measured by ImageJ software (NIH).

Cell viability assay. For all experiments, the cell viability was assessed using a tetrazolium-based assay (CellTiter 96 AQueous One solution - Promega Corporation, Madison, WI). Approximately 3000 cells in $50 \mu 1$ of media per well were plated in 96-well plates in triplicate. Twenty-four hours after plating, the cells were subjected to specific treatment regimens with the addition of $50 \mu \mathrm{l}$ of treatment media to achieve the prescribed treatment concentrations. DMSO, in equal amounts to the treatment conditions, was added to the media in the control condition. Once the treatment was complete, $20 \mu \mathrm{l}$ of the AQueous One solution was added to each well, for a final volume of $120 \mu 1$. Colorimetric analysis using a 96-well plate reader (Vmax Kinetic microplate reader, 
A

RT4

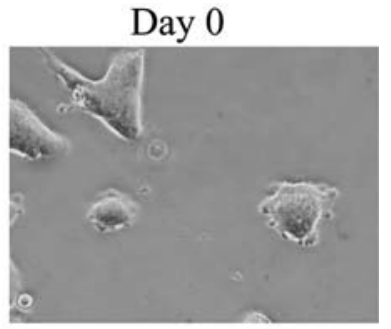

CRL1749

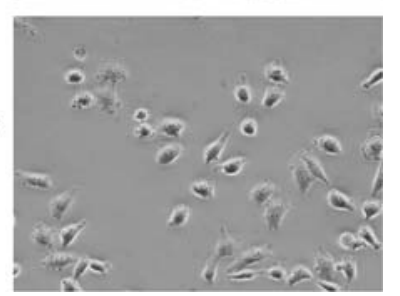

Figure 2A. RT4 and CRL1749 were serum-starved for $24 \mathrm{~h}$ before treatment.

Molecular Devices, Sunnyvale, CA) was performed between 1 and $4 \mathrm{~h}$ (wavelength of $490 \mathrm{~nm}$ ) after the addition of the AQueous One solution. Cell viability assays were performed in triplicate. Cell viability data and synergy analysis was performed with the CalcuSyn software program (Biosoft, Ferguson, MO; version 2, 2005) using a non-constant ratio analysis and statistical method described by Chou and Talalay to calculate the combination index (CI) values (21). The resulting $\mathrm{CI}$ is a quantitative measurement of the degree of interaction between different drugs used in combination. A CI value of 1 signifies an additive effect, a CI $>1$ denotes antagonism and a $\mathrm{CI}$ of $<1$ indicates synergy.

\section{Results}

Cell characterization based on E-cadherin and vimentin expression. RT4 and HTB3 cells exhibited epithelial-like cell features, including positive expression of E-cadherin without notable vimentin (Fig. 1). In contrast, the CRL1749 and J82 cells were negative for E-cadherin, but did show positive vimentin expression, consistent with a mesenchymal characterization. Based on these findings, RT4 and CRL1749 were selected to represent epithelial and mesenchymal cell types, respectively, for the subsequent experiments.

Morphological changes of RT4 and CRL1749 cells after treatment with EGF, VEGF, and vandetanib. RT4 cells tend to form tight clusters with closely apposed cell-to-cell interactions, typical of epithelial cells (Fig. 2A). After EGF treatment, the cells exhibited EMT morphological changes including increased intercellular separation with the development of pseudopodia, which occurred progressively over 3 days (Fig. 2B). These changes were assessed with multiple concentrations $(5,10,20,40 \mathrm{ng} / \mathrm{ml})$ of EGF, while the changes induced by $5-20 \mathrm{ng} / \mathrm{ml}$ EGF are similar but less prominent (not shown). The use of vandetanib blocked these changes. However, VEGF, at concentrations of (5 to $40 \mathrm{ng} / \mathrm{ml}$ ) did not seem to yield notable morphological alterations (Fig. 2B).

In contrast, CRL1749 cells grew in a scattered pattern and exhibited a spindle-like fibroblastoid cell shape typical of mesenchymal-type cells (Fig. 2A). After EGF or VEGF treatment, these cells did not demonstrate any significant change (Fig. 2C). However, after being cultured with vandetanib, the CRL1749 cells adopted a clustered or adhesive morphology, more typical of epithelial type cells, independent of the addition of EGF or VEGF.

Expression of EGFR, VEGFR-2, E-cadherin and vimentin after treatment. In the epithelial-like RT4 cells, expression of EGFR decreased with increasing concentrations of singleagent EGF or the combination of EGF plus vandetanib. There was no apparent change in expression of VEGFR-2, E-cadherin or vimentin with single-agent EGF treatment. In contrast, with vandetanib treatment, E-cadherin expression was substantially increased regardless of EGF (Fig. 3A). VEGFR-2 and vimentin expression was not affected by EGF or vandetanib treatment, either alone or in combination.

Similarly, treatment of RT4 cells with either VEGF alone or in combination treatment with vandetanib did not alter expression of vimentin, while the combination led to increased E-cadherin expression regardless of the presence of VEGF (Fig. 3B). EGFR expression was not substantially altered with VEGF alone or in combination with vandetanib. However, following treatment with vandetanib plus the higher doses of VEGF, the expression of VEGFR-2 decreased slightly.

In the mesenchymal-like CRL1749 cells, the expression of vimentin was consistently decreased after treatment with the combination of vandetanib plus either growth factor compared with EGF or VEGF alone (Fig. 3C and D). Inhibition of VEGFR and EGFR with vandetanib reduced EMT features in both cell types as measured by protein expression changes (Fig. 3A-D).

To summarize, in the epithelial-like RT4 cells, vandetanib treatment was associated with a significant increase in the E-cadherin expression, independent of EGF or VEGF administration (Fig. 3A and B). In the mesenchymal-like CRL1749 cells, vandetanib decreased the expression of vimentin, regardless of the addition of EGF or VEGF (Fig. 3C and D). Consistent with their baseline characteristics, the expression of vimentin in RT4 cells and E-cadherin in CRL1749 cells remained undetectable throughout these experiments.

The analysis of the expression of EGFR and VEGFR-2 yielded more complex findings. Vandetanib treatment decreased the expression of EGFR in RT4 cells when they were treated with EGF simultaneously, but not in CRL1749 cells. The expression of EGFR did not change in RT4 cells and showed a notable increase in CRL1749 cells when treated with the combination of vandetanib plus VEGF. The expression of VEGFR-2 decreased in RT4 cells when vandetanib was added to the EGF or VEGF treatment (Fig. 3A and B); in the CRL1749 cells, the VEGFR-2 expression was unchanged when treated with the combination of EGF and vandetanib, and increased with the combination of VEGF and vandetanib (Fig. 3C and D).

Cell viability with vandetanib and cisplatin treatment concurrently and sequentially. The epithelial and mesenchymal cell lines showed different degrees of viability when treated with vandetanib and cisplatin concurrently (Fig. 4A-D). Improved growth inhibition was observed with the drug 
B

Control

Day 2

Day 3
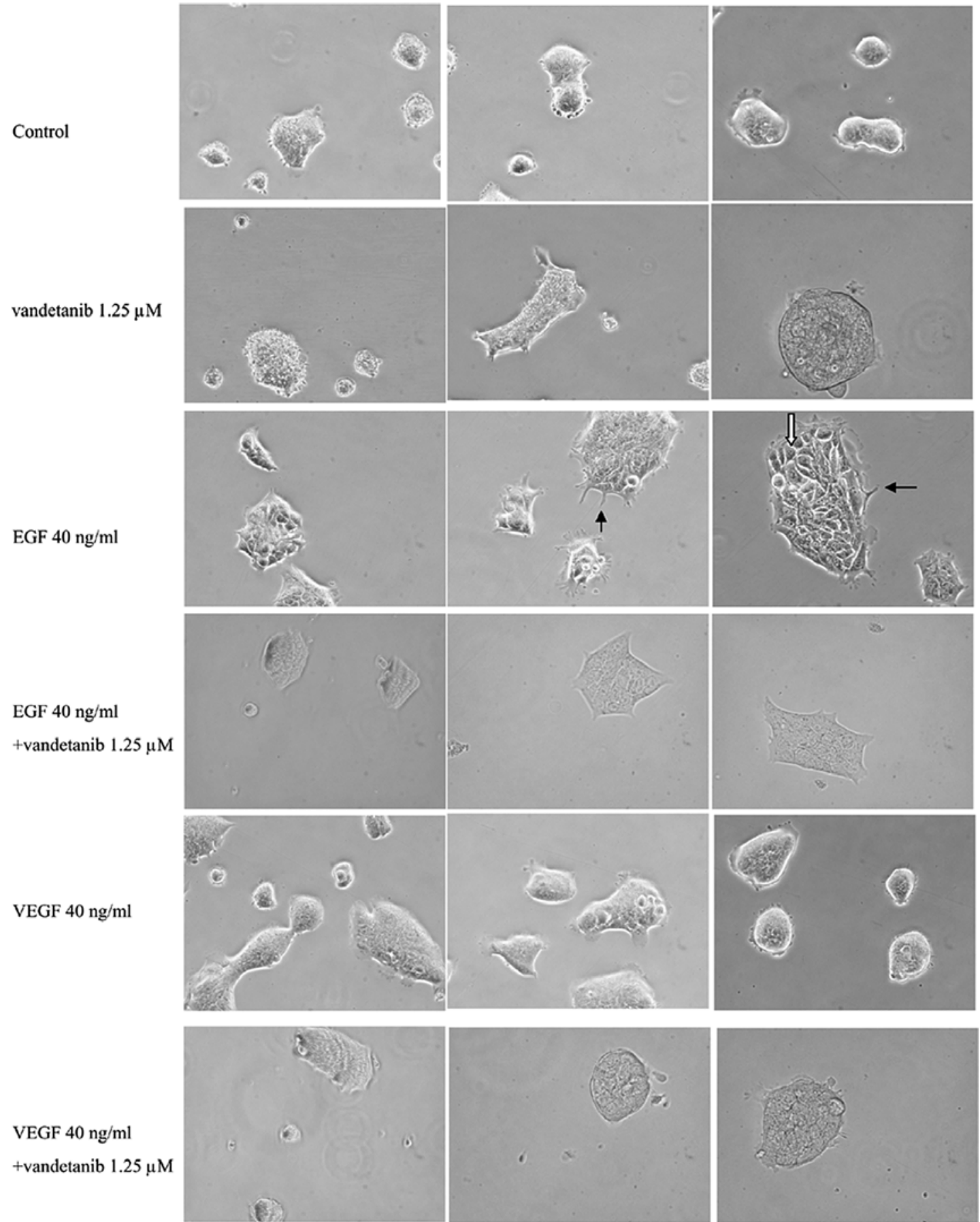

Figure 2B. RT4 cells were serum-starved $(0.5 \%)$ for $24 \mathrm{~h}$ before treated in the conditions indicated. On day 1,2 and 3 post treatment, the images were taken with Nikon light microscope separately. The images are representative ones in which concentration of both EGF and VEGF were 40 ng/ml. Solid arrows indicate pseudopodia of RT4 cells while the open arrow indicates the increasing intercellular separation.

combination in the epithelial RT4 and HTB3 cells, but not in the CRL1749 and J82 cells. Based on these findings, RT4 and CRL1749 cells were selected for sequential treatment testing, representing the epithelial and mesenchymal-type cells, respectively. Notably, the sequential treatment with vandetanib followed by cisplatin improved synergy in the CRL1749 cells (Fig. 4F) while the results with this sequential treatment did not appear to differ from the concurrent treatment in the RT4 cells (Fig. 4E). Vandetanib treatment therefore appeared to sensitize the CRL1749 cells to cisplatin treatment, when administered in a sequential manner. The CI values for these sequential treatment experiments demonstrate synergistic activity in both the RT4 cells (Fig. 5A) (CI range: 0.24-0.83) and CRL1749 cells (CI range: 0.66-1.08) (Fig. 5B). While both the sequential and concurrent experiments treated the cells for $96 \mathrm{~h}$, the absolute activity cannot be compared (e.g. Fig. 4A, C, E and F), since the cisplatin treatment exposure was $96 \mathrm{~h}$ in the concurrent, but only $48 \mathrm{~h}$ in the sequential experiments. 


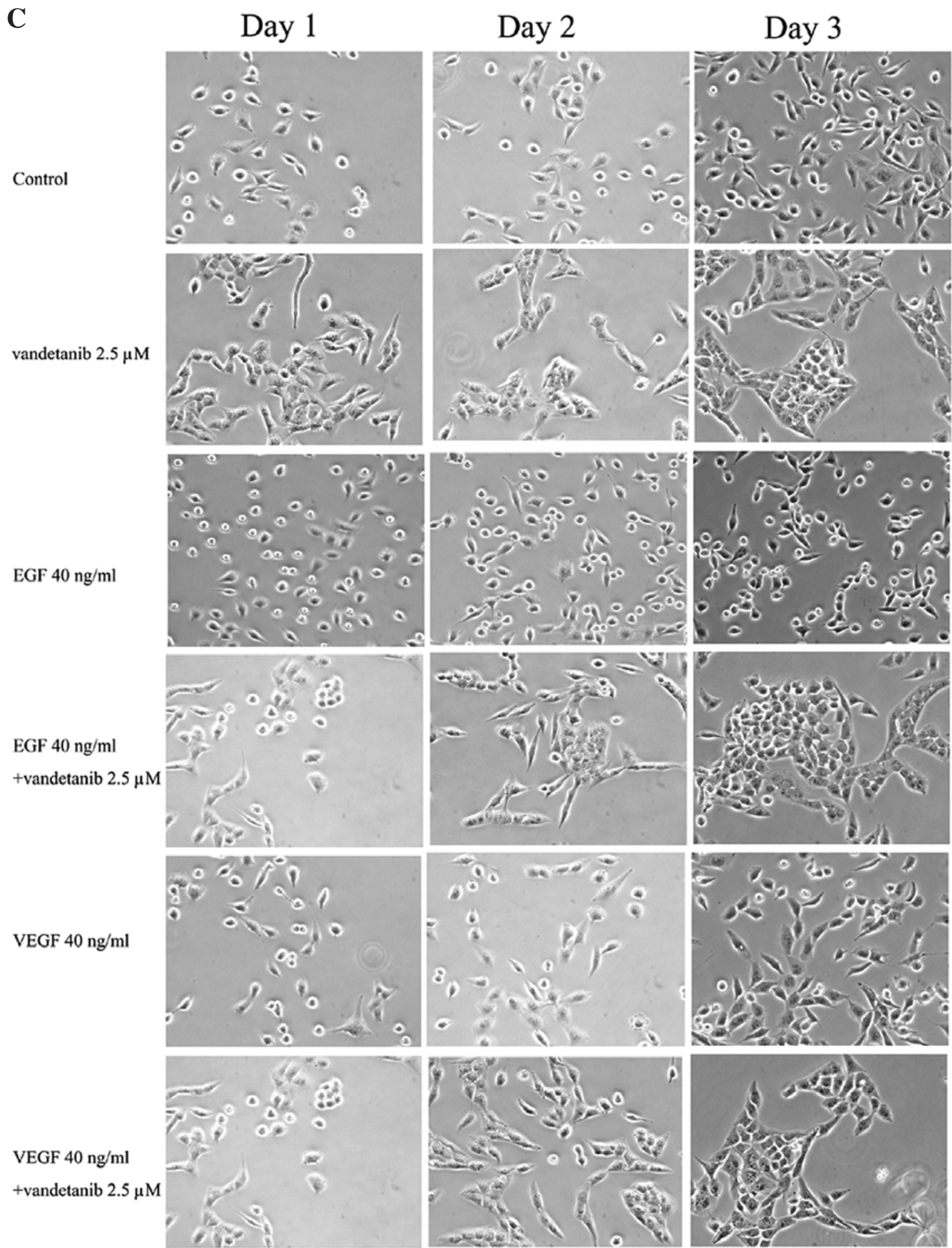

Figure 2C. CRL1749 cells were serum-starved (0.5\%) for $24 \mathrm{~h}$ before treated in the conditions indicated. On day 1,2 and 3 post treatment, the images were taken with Nikon light microscope separately. The images are representative ones in which concentration of both EGF and VEGF were 40 ng/ml. After treated with vandetanib, CRL1749 cells acquired epithelial-like features showing clustered growth pattern.

\section{Discussion}

In our previous study, we showed that vandetanib has a synergistic effect in combination with cisplatin in bladder cancer cells (20). In the current study, we demonstrate that the inhibitory effect of vandetanib is correlated with EMT, an important process in cancer progression and metastasis. Markers of EMT, including the loss of E-cadherin and gain of vimentin, correlate with laboratory and clinical assessments of tumor invasiveness $(22,23)$.
In this study, we found that exogenous EGF induced EMTlike morphological changes in the epithelial-like bladder cancer cell lines (RT4) (Fig. 2B). Although there were no apparent morphological changes in CRL1749 cells with EGF or VEGF treatment, the expression of vimentin increased in a dose-dependent manner with these growth factors, demonstrating an increased mesenchymal character (Figs. 2C; and $3 \mathrm{C}$ and D). Inhibition of VEGFR and EGFR with vandetanib decreased the mesenchymal features of the CRL1749 cells and enhanced the epithelial features of the RT4 
A

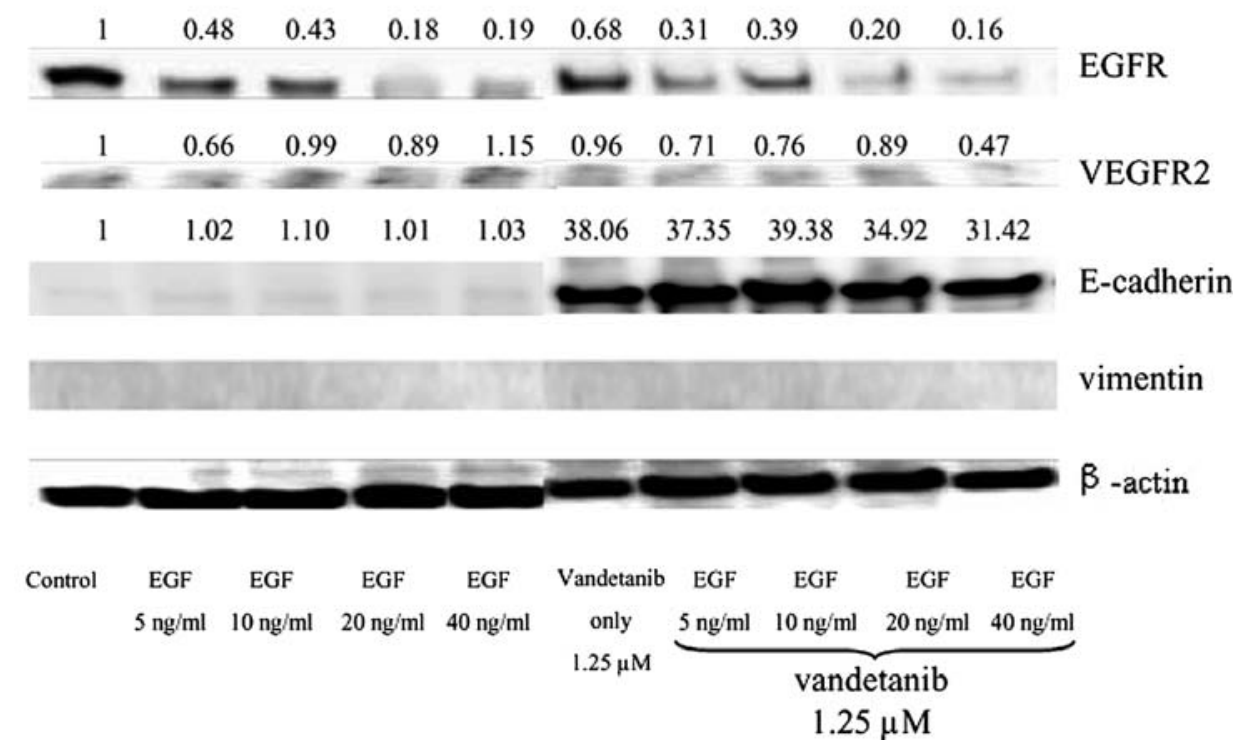

B

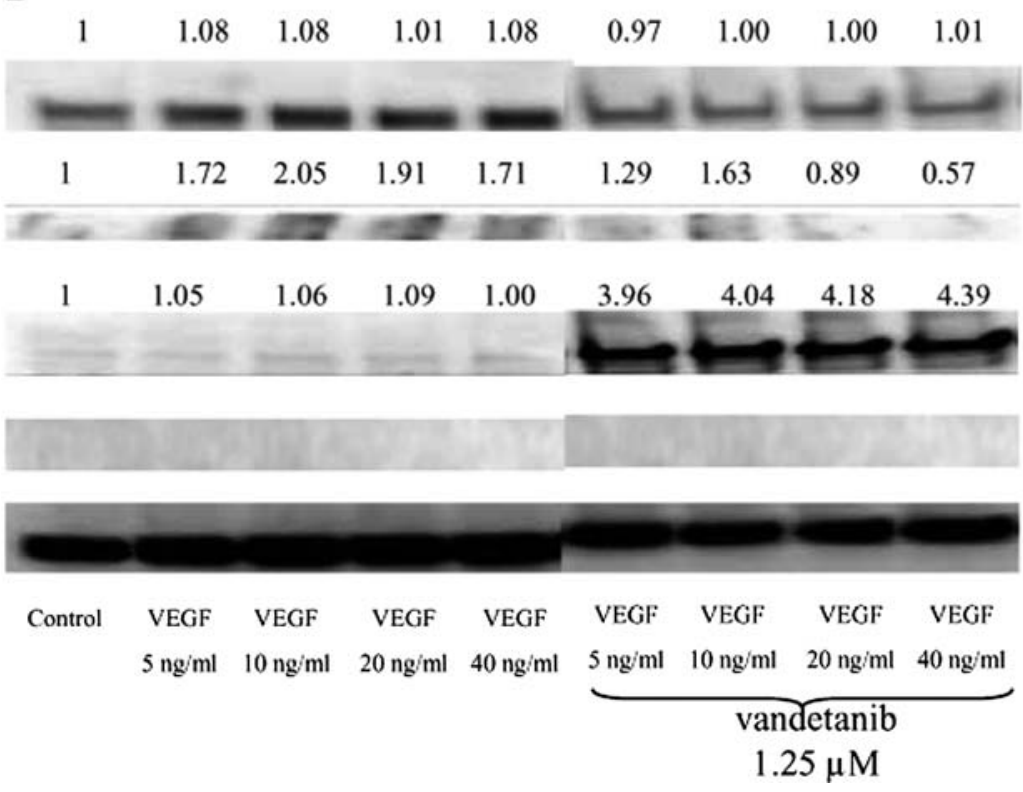

EGFR

VEGFR2

E-cadherin

vimentin

$\beta$-actin

C

\begin{tabular}{rrrrrrrrrrrr}
1 & 1.10 & 1.59 & 1.72 & 3.33 & 4.22 & 2.17 & 1.63 & 1.92 & 1.82 \\
1 & 1.49 & 1.50 & 1.38 & 2.00 & 1.60 & 1.13 & 1.21 & 1.80 & 1.40 \\
\hline & & & & & & & & &
\end{tabular}

EGFR

VEGFR2

E-cadherin

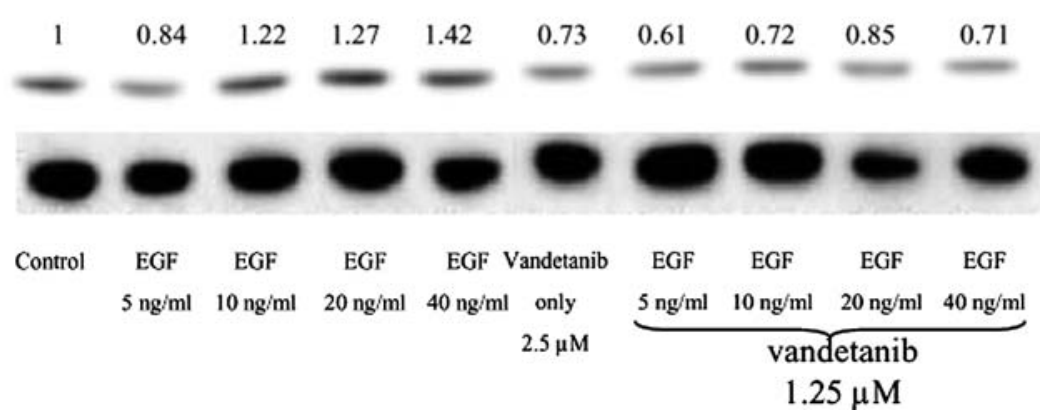

Vimentin

$\beta$-actin 


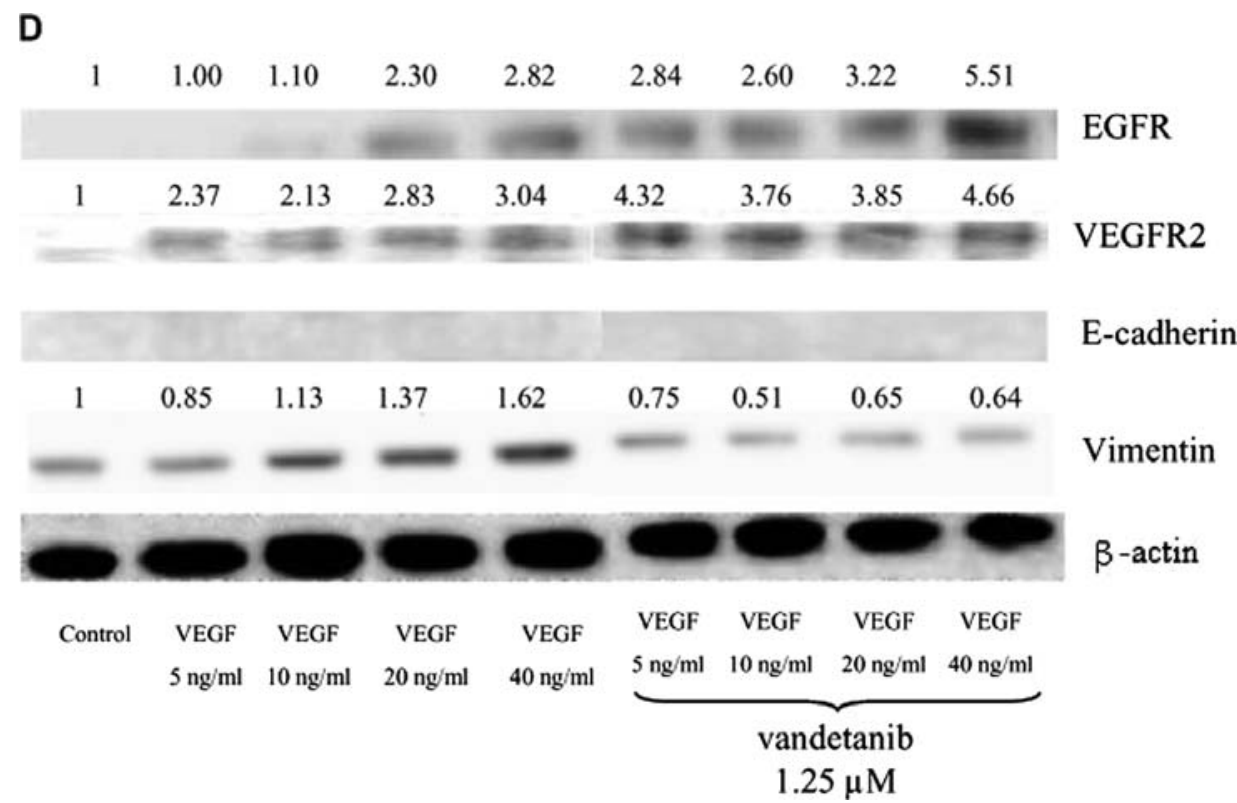

Figure 3. Protein expressions of human bladder cancer cells after treatment of EGF, VEGF, vandetanib or their combination. Cells were serum-starved for $24 \mathrm{~h}$ and EGF, VEGF, vandetanib or the combination were added into medium with $0.5 \%$ FBS. After 72 consecutive hours, the cells were harvested for Western blotting. The treatment medium was changed everyday. The numbers above the protein bands are the ratios of densities (measured by Image J software) of the examined protein to B-actin after normalized into the control. (A and B) RT4; (C and D) CRL1749.

cells (Fig. 3A-D). The baseline epithelial or mesenchymal nature of the bladder cancer cells predicted their sensitivity to concurrent cisplatin and vandetanib treatment (Fig. 4A-D). Importantly, sequential treatment with vandetanib followed by cisplatin appears to sensitize the mesenchymal cells to this chemotherapeutic combination, with synergy observed for the sequential combination but not for the concurrent treatment of these cells (Figs. 4E and F; and 5A and B).

Growth factor stimulation and microenvironment alterations appear to contribute to the molecular events associated with EMT during cancer progression $(8,24)$. The enhanced expression of several growth factors including EGF may result in an up-regulated expression of numerous gene products involved in the EMT process (24). Several groups have demonstrated EGF-induced EMT changes. Lo et al (13) found that chronic EGF exposure promoted EMT and TWIST gene expression in breast and pancreatic cancer cells, where TWIST serves as an EMT promoting transcription factor. In a bladder cancer model, Cattan et al (25) showed that CAL 29 cells expressed characteristic features of EMT after TGF- $\alpha$ and EGF treatment. Ahmed et al (26) reported that the combination of EGF and hydrocortisone induced EMT on the ovarian surface epithelium with characteristic cellular fibroblast-like morphology and growth pattern changes. Similar to the role of EGF in EMT, prolonged treatment with VEGF can also trigger EMT phenotypic changes in human pancreatic cell lines (14).

In the present study, the expression of vimentin in CRL1749 increased after treatment with EGF and VEGF, consistent with further EMT transition. Interestingly, both EGF and VEGF caused mesenchymal-morphological changes without any notable changes in E-cadherin expression in RT4 cells. While others have reported decreased E-cadherin and increased vimentin expression with VEGFR-1 activation in other non-bladder models (14), our results may differ since treatments were given under serum-starvation conditions, leading to low baseline levels of E-cadherin.

Many studies have focused on the relationship between EMT and tumor invasive and metastatic potential. Chemotherapy insensitivity is a major concern in the treatment of patients with advanced bladder cancer and it contributes to the poor prognosis of many of these patients. While metastases and chemoresistance are separate clinical events, there is evidence of a direct biologic connection between them (27). Kajiyama et al (27) described a possible link between paclitaxel resistance and the facilitated metastatic potential of epithelial ovarian carcinoma. Yang et al (28) found that induction of EMT may contribute to decreased efficacy of therapy in chemoresistant colorectal cancer, as the tumor cells switch from a proliferative to an invasive phenotype. Therefore, disrupting EMT may both limit the invasive potential of tumors and the development of chemotherapy resistance. There are several studies indicating that loss of E-cadherin is a marker of poor response to the anti-proliferative effect of EGFR inhibitors which include monoclonal antibodies (cetuximab) and tyrosine kinase inhibitors (erlotinib and gefitinib) against EGFR (23,29-31). Zhuo et al (32) showed TWIST depletion by RNA interference sensitized A549 lung cancer cells to cisplatin chemotherapy.

In the present study, RT4 and HTB3 cells showed different responsiveness to the concurrent treatment of vandetanib plus cisplatin compared with CRL1749 and J82 cells, predicted by the cell baseline epithelial or mesenchymal features. The mesenchymal CRL1749 cell resistance to cisplatin with concurrent vandetanib was diminished with vandetanib pretreatment. These results suggest that given the inhibition of 
A

RT4 V+C

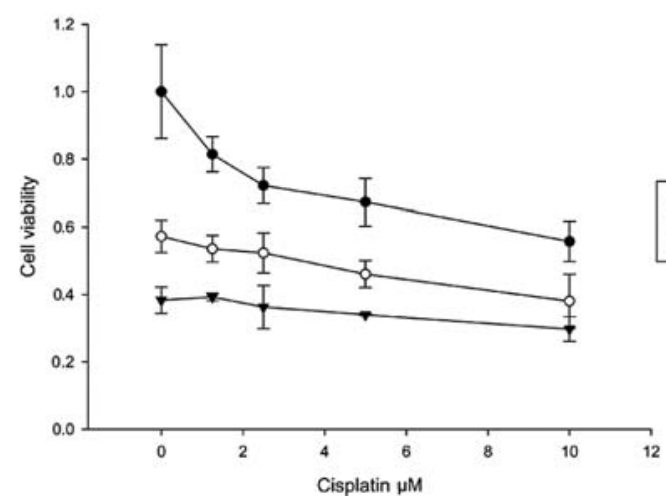

$\mathrm{C}$

CRL1749 V+C

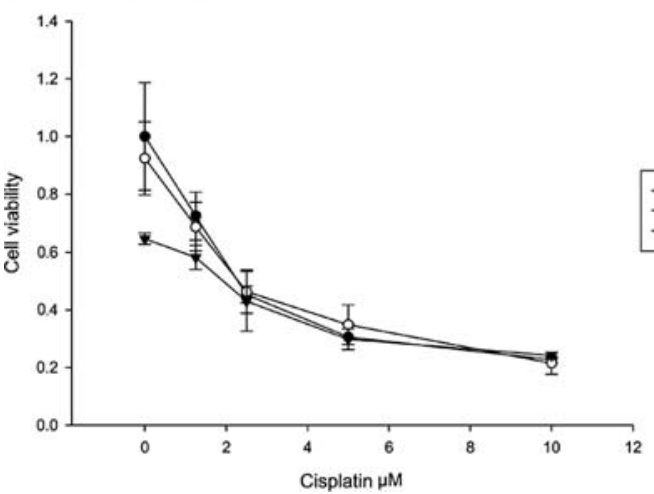

E

RT4 V $\rightarrow$ C

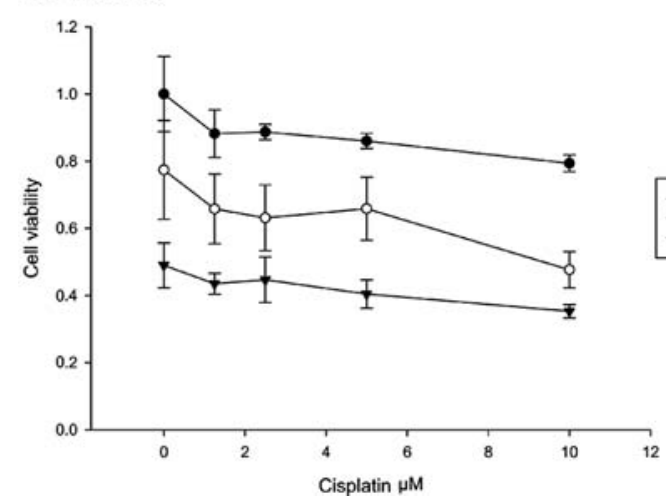

B

HTB3 V+C

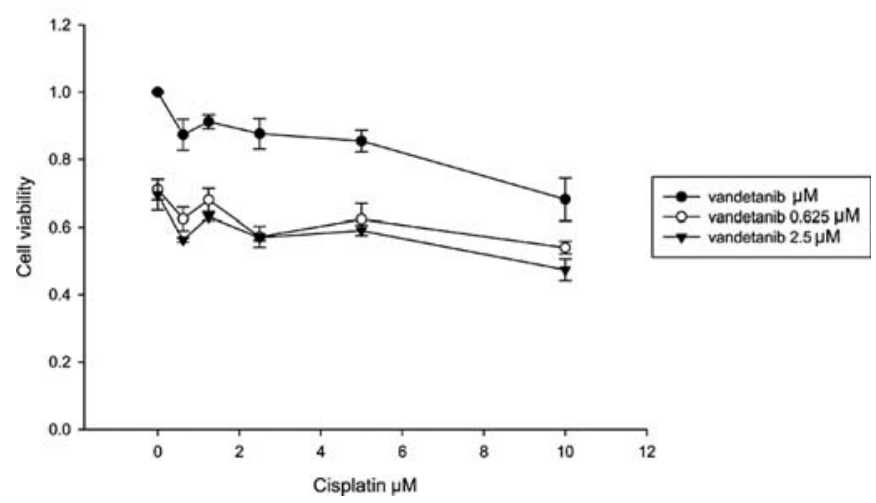

$\mathrm{D}$

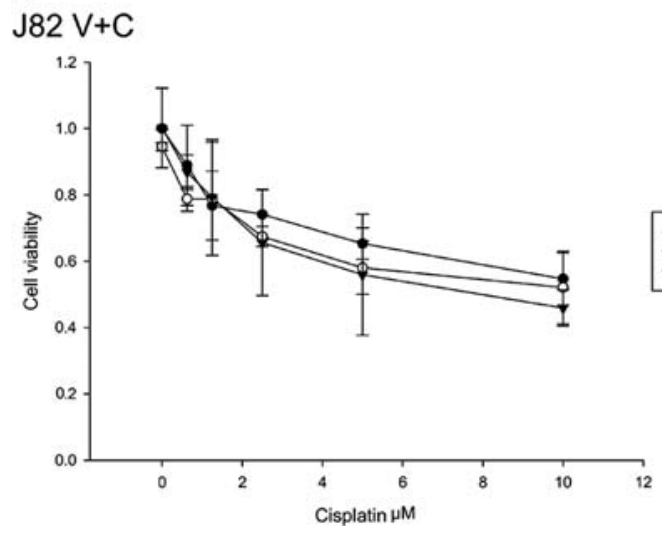

$\mathrm{F}$

CRL1749 V $\rightarrow C$

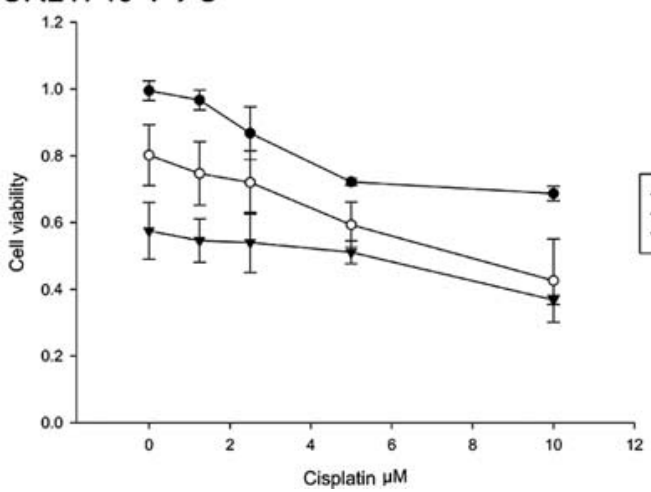

Figure 4. Cell viability results of concurrent treatment of vandetanib plus cisplatin for (A) RT4, (B) HTB3, (C) CRL1749 and (D) J82 cells are shown. The results of sequential treatment of vandetanib followed by cisplatin for (E) CRL1749 and (F) RT4 cells, are shown. The two drugs were added into the medium with $3.75 \%$ FBS.

both VEGFR and EGFR, the cells adopted a more epithelial character. Importantly, pre-treatment with vandetanib increased cisplatin sensitivity of these cells, with this sequential treatment approach demonstrating synergy in contrast to the concurrent approach. This suggests that blocking the effects of the growth factors associated with EMT may reverse the chemotherapy resistance seen in this setting.

The data from the present study do not show a clear relationship between the expression of VEGFR-2, EGFR, and treatment with vandetanib. One of the limitations in our work was the lack of examination of phosphorylation of VEGFR-2 and EGFR. These experiments were focused on the relationship of the growth factors EGF and VEGF with EMT, utilizing vandetanib to selectively inhibit VEGR and EGFR. Additional evaluation of the exact mechanism of these interactions, including the phosphorylation of these receptors, warrants further study. Selective EGFR inhibition, in comparison to the inhibition of both VEGFR/EGFR as evaluated herein, may also be examined in subsequent experiments to better determine the relative contribution of VEGFR and EGFR in this process. Another limitation in our 
A

\section{RT4 V $\rightarrow$ C}

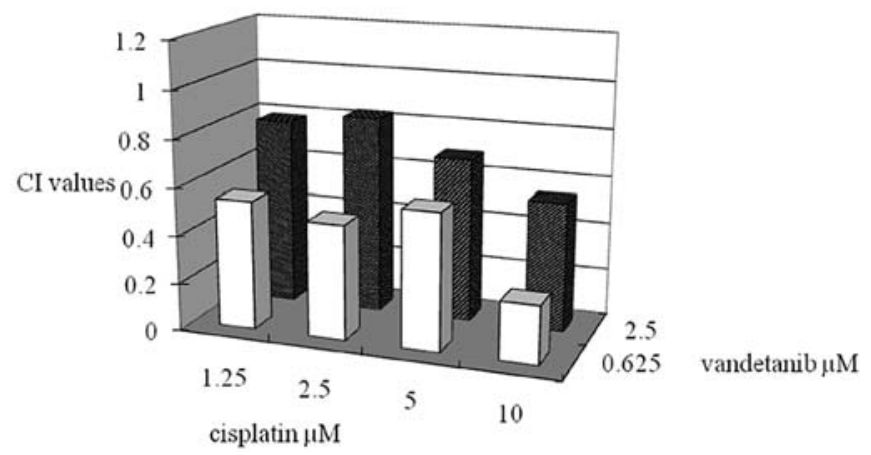

B

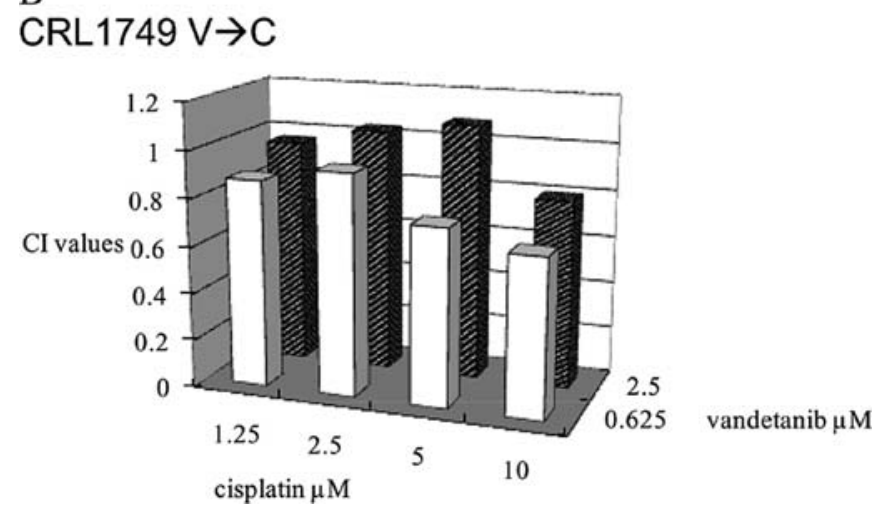

Figure 5. The combination index (CI) levels of sequential treatment of vandetanib followed by cisplatin for (A) RT4 and (B) CRL1749 cells.

work was the lack of fluorescent immunohistochemistry to evaluate the loss of polarity, which could have further confirmed EMT. We instead used morphological observations combined with protein characteristics to evaluate EMT changes in the cells. There are several isoforms of VEGFR. In present study, only VEGFR-2 was investigated. Other VEGFRs which are implicated in tumor responses (notably VEGFR-1) were not examined.

In conclusion, exogenous VEGF and EGF can cause morphologic changes as well as E-cadherin and vimentin protein expression changes in bladder cancer cells, consistent with EMT. VEGFR and EGFR inhibition via vandetanib increases the epithelial nature of bladder cancer cells with increased E-cadherin and decreased vimentin expression. The epithelial-like bladder cancer cells showed improved response to cisplatin with concurrent vandetanib, but mesenchymallike cells did not. Sequential therapy with vandetanib followed by cisplatin demonstrates improved anti-proliferative activity in mesenchymal cells, suggesting a role for inhibiting both VEGFR and EGFR in patients with mesenchymal tumor characteristics prior to traditional chemotherapy.

\section{Acknowledgements}

This study was supported in part by a Paul Calabresi K12 clinical scholar grant (T.W.F.) awarded to the University of Colorado Denver (K12CA086913). Vandetanib used in these studies was supplied by AstraZeneca Pharmaceuticals, Macclesfield, UK.

\section{References}

1. Jemal A, Siegel R, Ward E, Hao Y, Xu J, Murray T and Thun MJ: Cancer statistics, 2008. CA Cancer J Clin 58: 71-96, 2008.

2. von der Maase H, Sengelov L, Roberts JT, et al: Long-term survival results of a randomized trial comparing gemcitabine plus cisplatin, with methotrexate, vinblastine, doxorubicin, plus cisplatin in patients with bladder cancer. J Clin Oncol 23: 4602-4608, 2005.

3. Savagner P, Boyer B, Valles AM, Jouanneau J and Thiery JP: Modulations of the epithelial phenotype during embryogenesis and cancer progression. Cancer Treat Res 71: 229-249, 1994.

4. Hay ED: An overview of epithelio-mesenchymal transformation. Acta Anat 154: 8-20, 1995.
5. Shook D and Keller R: Mechanisms, mechanics and function of epithelial-mesenchymal transitions in early development. Mech Dev 120: 1351-1383, 2003.

6. Huber MA, Azoitei N, Baumann B, et al: NF-kappaB is essential for epithelial-mesenchymal transition and metastasis in a model of breast cancer progression. J Clin Invest 114: 569-581, 2004.

7. Grünert $\mathrm{S}$, Jechlinger $\mathrm{M}$ and Beug $\mathrm{H}$ : Diverse cellular and molecular mechanisms contribute to epithelial plasticity and metastasis. Nat Rev Mol Cell Biol 4: 657-665, 2003.

8. Thiery JP: Epithelial-mesenchymal transitions in tumour progression. Nat Rev Cancer 2: 442-454, 2002.

9. Chaffer CL, Brennan JP, Slavin JL, Blick T, Thompson EW and Williams ED: Mesenchymal-to-epithelial transition facilitates bladder cancer metastasis: role of fibroblast growth factor receptor-2. Cancer Res 66: 11271-11278, 2006.

10. Nutt JE, Foster PA, Mellon JK and Lunec J: hEGR1 is induced by EGF, inhibited by gefitinib in bladder cell lines and related to EGF receptor levels in bladder tumours. Br J Cancer 96: 762-768, 2007.

11. Xia G, Kumar SR, Hawes D, et al: Expression and significance of vascular endothelial growth factor receptor 2 in bladder cancer. J Urol 175: 1245-1252, 2006.

12. Sabbah M, Emami S, Redeuilh G, et al: Molecular signature and therapeutic perspective of the epithelial-to-mesenchymal transitions in epithelial cancers. Drug Resist Updat 11: 123-151, 2008.

13. Lo HW, Hsu SC, Xia W, et al: Epidermal growth factor receptor cooperates with signal transducer and activator of transcription 3 to induce epithelial-mesenchymal transition in cancer cells via up-regulation of TWIST gene expression. Cancer Res 67: 9066-9076, 2007.

14. Yang AD, Camp ER, Fan F, et al: Vascular endothelial growth factor receptor-1 activation mediates epithelial to mesenchymal transition in human pancreatic carcinoma cells. Cancer Res 66: 46-51, 2006.

15. Min C, Eddy SF, Sherr DH and Sonenshein GE: NF-kappaB and epithelial to mesenchymal transition of cancer. J Cell Biochem 104: 733-744, 2008.

16. Lamouille $\mathrm{S}$ and Derynck R: Cell size and invasion in TGFbeta-induced epithelial to mesenchymal transition is regulated by activation of the mTOR pathway. J Cell Biol 178: 437-451, 2007.

17. Al-Nedawi K, Meehan B, Micallef J, Lhotak V, May L, Guha A and Rak J: Intercellular transfer of the oncogenic receptor EGFRvIII by microvesicles derived from tumour cells. Nat Cell Biol 10: 619-624, 2008.

18. Dissanayake SK, Wade M, Johnson CE, et al: The Wnt5A/ protein kinase $\mathrm{C}$ pathway mediates motility in melanoma cells via the inhibition of metastasis suppressors and initiation of an epithelial to mesenchymal transition. J Biol Chem 282: 17259-17271, 2007.

19. Wedge SR, Ogilvie DJ, Dukes M, et al: ZD6474 inhibits vascular endothelial growth factor signaling, angiogenesis, and tumor growth following oral administration. Cancer Res 62: 4645-4655, 2002. 
20. Flaig TW, Su LJ, McCoach C, Li Y, Raben D, Varella-Garcia M and Bemis LT: Dual epidermal growth factor receptor and vascular endothelial growth factor receptor inhibition with vandetanib sensitizes bladder cancer cells to cisplatin in a doseand sequence-dependent manner. BJU Int 103: 1729-17237, 2009.

21. Chou TC and Talalay P: Quantitative analysis of dose-effect relationships: the combined effects of multiple drugs or enzyme inhibitors. Adv Enzyme Regul 22: 27-55, 1984.

22. Willipinski-Stapelfeldt B, Riethdorf S, Assmann V, et al: Changes in cytoskeletal protein composition indicative of an epithelial-mesenchymal transition in human micrometastatic and primary breast carcinoma cells. Clin Cancer Res 11: 8006-8014, 2005.

23. Buck E, Eyzaguirre A, Barr S, et al: Loss of homotypic cell adhesion by epithelial-mesenchymal transition or mutation limits sensitivity to epidermal growth factor receptor inhibition. Mol Cancer Ther 6: 532-541, 2007.

24. Mimeault M and Batra SK: Interplay of distinct growth factors during epithelial mesenchymal transition of cancer progenitor cells and molecular targeting as novel cancer therapies. Ann Oncol 18: 1605-1619, 2007.

25. Cattan N, Rochet N, Mazeau C, et al: Establishment of two new human bladder carcinoma cell lines, CAL 29 and CAL 185. Comparative study of cell scattering and epithelial to mesenchyme transition induced by growth factors. Br J Cancer 85: 1412-1417, 2001.

26. Ahmed N, Maines-Bandiera S, Quinn MA, Unger WG, Dedhar S and Auersperg N: Molecular pathways regulating EGF-induced epithelio-mesenchymal transition in human ovarian surface epithelium. Am J Physiol Cell Physiol 290: C1532-C1542, 2006.
27. Kajiyama H, Shibata K, Terauchi M, Yamashita M, Ino K, Nawa A and Kikkawa F: Chemoresistance to paclitaxel induces epithelial-mesenchymal transition and enhances metastatic potential for epithelial ovarian carcinoma cells. Int J Oncol 31: 277-283, 2007.

28. Yang AD, Fan F, Camp ER, et al: Chronic oxaliplatin resistance induces epithelial-to-mesenchymal transition in colorectal cancer cell lines. Clin Cancer Res 12: 4147-4153, 2006.

29. Black PC, Brown GA, Inamoto T, et al: Sensitivity to epidermal growth factor receptor inhibitor requires E-cadherin expression in urothelial carcinoma cells. Clin Cancer Res 14: 1478-1486, 2008.

30. Yauch RL, Januario T, Eberhard DA, et al: Epithelial versus mesenchymal phenotype determines in vitro sensitivity and predicts clinical activity of erlotinib in lung cancer patients. Clin Cancer Res 11: 8686-8698, 2005.

31. Witta SE, Gemmill RM, Hirsch FR, et al: Restoring E-cadherin expression increases sensitivity to epidermal growth factor receptor inhibitors in lung cancer cell lines. Cancer Res 66: 944-950, 2006

32. Zhuo WL, Wang Y, Zhuo XL, Zhang YS and Chen ZT: Short interfering RNA directed against TWIST, a novel zinc finger transcription factor, increases A549 cell sensitivity to cisplatin via MAPK/mitochondrial pathway. Biochem Biophys Res Commun 369: 1098-1102, 2008 